\title{
Small-signal theory of a grating-based free-electron laser in three dimensions
}

\author{
J. D. Jarvis, H. L. Andrews, and C. A. Brau \\ Department of Physics and Astronomy, Vanderbilt University, Nashville, Tennessee 37235, USA
}

(Received 28 October 2008; published 3 February 2010)

\begin{abstract}
We present an analytic theory for the small-signal operation of a grating-based free-electron laser that includes the effects of transverse diffraction on the evanescent wave. In this device, the electron beam interacts with an evanescent wave of the grating that bunches the beam and creates superradiant SmithPurcell radiation. We find that the evanescent wave is guided by the electron beam, giving an optical-mode width that depends on the gain. We consider the cases of very wide and very narrow electron beams. For a wide electron beam, the cubic dispersion relation previously found for slow-wave structures is recovered. When the electron beam is narrow, so that gain guiding is important, a fifth-order dispersion relation is found instead. Diffraction in a system where the group velocity is very different (sometimes negative) from the phase velocity leads to unexpected results. The Brillouin zone subdivides into four regions; only two physically allowed (gain-guided) roots are obtained in the regions near the center of the Brillouin zone, but three are found in the regions away from the center. In the left half of the Brillouin zone, corresponding to high electron energy, the device operates on a convective instability, as an amplifier. In the right half of the Brillouin zone, where the group velocity is negative, the device operates on an absolute instability, as an oscillator. In the region where only two guided modes exist, oscillator operation will be more difficult.
\end{abstract}

DOI: 10.1103/PhysRevSTAB.13.020701

PACS numbers: 41.60.Cr, 52.59.Rz

\section{INTRODUCTION}

The wide range of potential applications for $\mathrm{THz}$ radiation is currently driving interest in the development of intense, compact, tunable THz sources. Such applications include resonant excitation and spectral analysis of chemical and biological molecules and systems, medical and industrial imaging, and investigations in materials science and nanostructures [1,2]. Electron beam-based devices are very promising sources of $\mathrm{THz}$ radiation. These include synchrotrons [3,4], conventional free-electron lasers [5-7], and slow-wave devices, such as traveling-wave tubes [8], orotrons [9], and backward-wave oscillators (BWOs) [10]. While synchrotrons and conventional FELs are large and expensive, slow-wave devices can be compact, laboratoryscale instruments. Commercially available BWOs, for example, produce about $1 \mathrm{~mW}$ at $1 \mathrm{THz}$, and weigh about $15 \mathrm{~kg}$ [11].

Slow-wave structures support subluminal electromagnetic modes, which may be driven by an electron beam passing in close proximity. This interaction causes amplitude growth of the subluminal optical wave and bunching in the electron beam. In a BWO, the group velocity of the optical wave is opposite that of the electron beam. This provides an intrinsic feedback mechanism, called an absolute instability, which causes the wave to oscillate without external feedback. In addition, when the slow-wave structure consists of an open diffraction grating, spontaneous Smith-Purcell radiation is created as the electrons pass close over the grating in a direction normal to the grooves [12-15]. When the electrons are bunched, the Smith-
Purcell radiation becomes coherent and much more intense [16-19]. When the bunching is periodic, the spectrum is altered and the Smith-Purcell radiation becomes superradiant [20], and intense radiation is produced at harmonics of the bunching frequency. When the periodic bunching is caused by interaction with an evanescent wave of the grating, intense radiation may be extracted at harmonics of the evanescent wave [21-25]. In addition, radiation at the frequency of the evanescent wave appears when the evanescent wave scatters off the ends of the grating. The grating-based FEL may be operated as an amplifier (convective instability), or as an oscillator (absolute instability), depending on the sign of the evanescent wave's group velocity.

In 1998, Walsh's group at Dartmouth passed the electron beam from a cast-off electron microscope over a small grating and observed $\mathrm{THz}$ Smith-Purcell radiation that became superradiant at currents above about $1 \mathrm{~mA}$ [26]. This generated considerable excitement as a $\mathrm{THz}$ source [27], and was followed by several other experiments by Walsh and others $[28,29]$. It was thought that the observed superradiance was caused by oscillation of an evanescent wave of the grating, and several theories seemed to show that the electron beam current used in the experiments was close to the threshold for supporting oscillation [30-34]. However, there was no evidence of the evanescent wave in the experiments, and no clear indication of the spectral changes that would be expected if the evanescent wave were oscillating. It seems now that the $2 \mathrm{D}$ theories used at that time were inadequate to describe the operation of those experiments, and the $3 \mathrm{D}$ theory presented here predicts that 
the experiments were more than an order of magnitude below the threshold for oscillation. The source of the superradiance observed in those experiments and in experiments performed more recently [35] lies elsewhere.

Three-dimensional PIC simulations have recently been performed for gratings with and without sidewalls at the ends of the grooves [36,37], and a 3D, small-signal theory for a device with sidewalls has recently been published [38]. However, it has not been possible numerically to treat gratings that are much wider than the electron beam, such as those used in the experiments described above. Kumar and Kim [39] consider analytically the propagation of an evanescent wave over the surface of an infinitely wide grating, including the effects of diffraction in the direction transverse to the electron beam, parallel to the grooves. However, they do not include the effects of the electron beam on the diffraction. They find that in the absence of the electron beam, the width of an evanescent wave focused in the transverse direction with a Rayleigh range $Z_{R}$ is $\sqrt{\lambda Z_{R} / 4 \pi \beta_{g}}$, where $\lambda$ is the free-space wavelength and $\beta_{g}$ is the group velocity of the wave. This width diverges near the Bragg point, where the group velocity vanishes, which illustrates the importance of guiding by the electron beam.

In this work, we analyze the effects of transverse diffraction in the optical beam of an infinitely wide grating FEL and include the effects of the electron beam. As in conventional free-electron lasers [40,41], the gain and the index of refraction of the electron beam act in the manner of a weak optical fiber to guide the optical beam. The approach used here is similar to that used for the 3D theory of the Cerenkov FEL [42]. As expected, three-dimensional effects decrease the gain substantially. Furthermore, compared to the 2D theory, the dependence of the gain on beam current increases due to gain guiding. For a wide electron beam, the cubic dispersion relation obtained in previous analyses of slow-wave structures $[8,43]$ is recovered. However, when the electron beam is narrow, gain guiding results in a fifth-order dispersion relation instead of the usual cubic dispersion relation, with unexpected results. We find that diffraction of the optical beam subdivides the Brillouin zone of the grating into two amplifier regions and two oscillator regions. For the amplifier and oscillator regions furthest from the Bragg point, we find that a fast wave is included in the physically allowed (gain-guided) solutions. This is surprising, considering the nature of a guided system. For the oscillator region closest to the Bragg point there are only two physically allowed solutions, so oscillation in this region of the Brillouin zone will be more difficult.

\section{DISPERSION}

In a grating FEL, resonant energy exchange between the electron beam and bound surface modes gives rise to
TABLE I. Example grating and beam parameters.
Groove width

Groove depth

Grating length

E-beam width/height

E-beam current

E-beam centroid height from grating top
Grating period
$173 \mu \mathrm{m}$ $62 \mu \mathrm{m}$ $100 \mu \mathrm{m}$ $12.7 \mathrm{~mm}$ $60 \mu \mathrm{m}$

$1 \mathrm{~mA}$

$30 \mu \mathrm{m}$ spatial modulations in the beam density. For an electron beam energy of $150 \mathrm{kV}$ and the grating parameters of Table I, the intensity scale height of the evanescent wave is $\Delta x=\beta \gamma \lambda / 4 \pi \approx 38 \mu \mathrm{m}$, where $\beta \sim 0.63$ is the normalized electron velocity, $\gamma=1 / \sqrt{1-\beta^{2}} \sim 1.3$ is the Lorentz factor, and $\lambda \sim 580 \mu \mathrm{m}$ is the free-space wavelength. We anticipate from simple diffraction arguments that the transverse mode width in this example is of order $\Delta y \approx \sqrt{\beta \lambda Z_{g} / 2 \pi}$, where $Z_{g}$ is the gain length. For a gain length on the order of the grating length, the transverse width is on the order of millimeters. Schematics of the device geometry with all pertinent dimensions are given in Figs. 1 and 2. Because the fields vanish exponentially above the scale height, we simplify the theory by allowing the electron beam to extend to infinity in the $x$ direction. A filling factor can be used to correct for errors introduced by this approximation [34].

In the following analysis we calculate the fields subject to the Maxwell equations and boundary conditions and solve for the dispersion relation. We treat the electron beam as a perturbation and calculate the resulting wave number and frequency shifts for solutions to the dispersion relation. The relative importance of the perturbation is on the order of $\omega_{e}^{2} / \omega^{2} \sim 10^{-5}$, where $\omega_{e}$ is the plasma frequency of the electron beam and $\omega$ is the radian frequency of the evanescent wave, so the perturbation is small. We assume in the analysis that the evanescent waves are pure transverse-magnetic (TM) modes of the grating. The empty grating of infinite width does not support transverse elec-

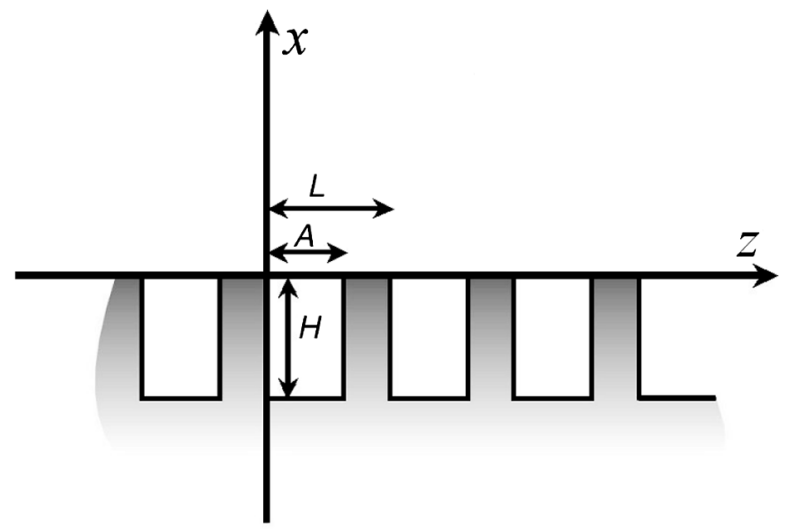

FIG. 1. Geometry of grating structure, viewed from the side. 


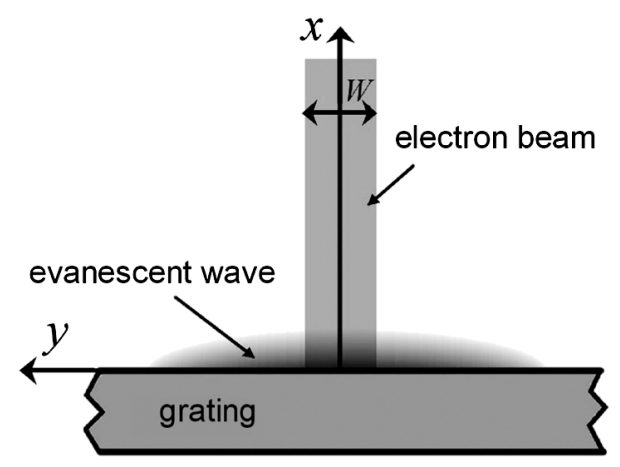

FIG. 2. Geometry of evanescent wave and electron beam, viewed from in front of the beam.

tric (TE) modes, so longitudinal magnetic fields arise only as a perturbation and may be ignored to lowest order. The accuracy of this approximation is confirmed by comparison of the predicted results with experiments and simulations. The frequencies of TM modes predicted when sidewalls are included [38] are in excellent agreement with those observed in experiments using a grating with sidewalls [35]. Similarly, comparison of the frequencies predicted for TM modes with those found in PIC simulations with sidewalls [36] shows excellent agreement. In the absence of sidewalls, there are no true TM modes of a finite grating. However, one might expect the modes of a grating that is wide compared to the scale height of the evanescent wave to be nearly TM modes. In recent simulations for a wide grating without sidewalls [44], it is found that highfrequency, mixed modes can be excited by a small antenna, but the frequencies of the lowest-order modes, those excited by an electron beam, are in excellent agreement with the frequencies of the TM modes of an infinite grating.

We begin by expressing the fields inside the grooves as Fourier series

$$
\begin{aligned}
E_{z}^{(g)} & =\sum_{n=0}^{\infty} E_{n}^{(g)}(x, y) \cos \left(\frac{n \pi}{A} z\right) e^{-i \omega t} \\
H_{y}^{(g)} & =\sum_{n=0}^{\infty} H_{n}^{(g)}(x, y) \cos \left(\frac{n \pi}{A} z\right) e^{-i \omega t},
\end{aligned}
$$

where $A$ is the groove width, and $\omega$ is the frequency. Each term in the fields must satisfy the wave equation, which is given in the grooves by

$$
\left[\nabla_{t}^{2}-\frac{n^{2} \pi^{2}}{A^{2}}+\frac{\omega^{2}}{c^{2}}\right] E_{n}^{(g)}(x, y)=0
$$

where $\nabla_{t}$ is the transverse gradient operator. Taking the Fourier transform of (3) in the $y$ direction we have

$$
\left[\frac{\partial^{2}}{\partial x^{2}}-k_{y}^{2}-\frac{n^{2} \pi^{2}}{A^{2}}+\frac{\omega^{2}}{c^{2}}\right] \tilde{E}_{n}^{(g)}\left(x, k_{y}\right)=0,
$$

where $k_{y}$ is the wave number in the $y$ direction. The solution for $\tilde{E}_{n}^{(g)}\left(x, k_{y}\right)$ that vanishes at the bottom of the grooves $(x=-H)$ is

$$
\tilde{E}_{n}^{(g)}\left(x, k_{y}\right)=\overline{\tilde{E}}_{n}^{(g)}\left(k_{y}\right) \sinh \left[\kappa_{n}\left(k_{y}\right)(x+H)\right],
$$

where

$$
\kappa_{n}^{2}=k_{y}^{2}+\frac{n^{2} \pi^{2}}{A^{2}}-\frac{\omega^{2}}{c^{2}} .
$$

For a TM mode, the components $\tilde{E}_{n}^{(g)}\left(x, k_{y}\right)$ and $\tilde{H}_{n}^{(g)}\left(x, k_{y}\right)$ are related through the Maxwell equations by

$$
\tilde{H}_{n}^{(g)}\left(x, k_{y}\right)=\frac{i \omega \varepsilon_{0} c^{2}}{\omega^{2}-\frac{n^{2} \pi^{2}}{A^{2}} c^{2}} \frac{\partial}{\partial x} \tilde{E}_{n}^{(g)}\left(x, k_{y}\right) .
$$

Substituting the solution for $\tilde{E}_{n}^{(g)}\left(x, k_{y}\right)$ into (7), we get

$$
\tilde{H}_{n}^{(g)}\left(x, k_{y}\right)=\overline{\tilde{H}}_{n}^{(g)}\left(k_{y}\right) \cosh \left[\kappa_{n}\left(k_{y}\right)(x+H)\right],
$$

where

$$
\overline{\tilde{H}}_{n}^{(g)}\left(k_{y}\right)=\frac{i \omega \varepsilon_{0} c^{2}}{\omega^{2}-\frac{n^{2} \pi^{2}}{A^{2}} c^{2}} \kappa_{n}\left(k_{y}\right) \overline{\tilde{E}}_{n}^{(g)}\left(k_{y}\right) .
$$

Above the grating we expand the fields in Floquet series (space harmonics) of the form

$$
\begin{gathered}
E_{z}^{(e)}=\sum_{r=-\infty}^{\infty} E_{r}^{(e)}(x, y) e^{i r K z} e^{i(k z-\omega t)} \\
H_{y}^{(e)}=\sum_{r=-\infty}^{\infty} H_{r}^{(e)}(x, y) e^{i r K z} e^{i(k z-\omega t)},
\end{gathered}
$$

where $k$ is the longitudinal wave number, $K=2 \pi / L$ is the grating wave number, and $L$ is the grating period. The electron beam is treated as an isotropic dielectric in its rest frame (primed coordinates), having an index of refraction given by [45]

$$
n^{\prime}\left(\omega^{\prime}\right)^{2}=1+\chi_{e}^{\prime}\left(\omega^{\prime}\right)=1-\frac{\omega_{e}^{\prime 2}}{\omega^{\prime 2}}
$$

where $\chi_{e}^{\prime}=-\left(\omega_{e}^{\prime 2} / \omega^{\prime 2}\right)$ is the frequency-dependent susceptibility, and $\omega_{e}^{\prime}$ is the plasma frequency in the rest frame. This approach is valid in the small-signal regime, where the electron beam can be treated as a linear dielectric. In adopting this approach, we implicitly ignore the temperature, or other sources of energy spread, of the electron beam. This approximation is valid provided that the relative transit time of two electrons along the length of the grating is small compared to the period of oscillation, that is, $|\Delta v / v|<1 / \omega T$, where $T=L / v$ is the transit time, $L$ is the grating length, $v$ is the electron velocity, and $\Delta v$ is the velocity spread. Using a more elaborate analysis, Kumar and Kim arrive at the same result [46]. For the example in Table I, with frequency $\omega \sim 3 \times$ $10^{12}$ radians $/ \mathrm{s}$, and velocity $v \sim 10^{8} \mathrm{~m} / \mathrm{s}$, the tolerable velocity spread is on the order of $0.3 \%$. For electrostatic 
acceleration, the velocity spread is typically much smaller than this.

In each region where the electron beam is uniform, the wave equation above the grating is then

$$
\left[\nabla_{t}^{\prime 2}-\left(k^{\prime}+r K^{\prime}\right)^{2}+\frac{\omega_{r}^{\prime 2}}{c^{2}}-\frac{\omega_{e}^{\prime 2}}{c^{2}}\right] E_{r}^{(e) \prime}\left(x^{\prime}, y^{\prime}\right)=0 .
$$

In terms of rest-frame variables, the frequency $\omega_{r}^{\prime}$ depends on the space harmonic, as denoted by the subscript $r$. We note that $(k+r K)^{2}-\left(\omega_{r}^{2} / c^{2}\right), E_{r}^{(e)}$, and the transverse dimensions, $x$ and $y$, are Lorentz invariant. Using the parameters of Table I, the plasma frequency in the lab frame is calculated to be of the order $\omega_{e} \sim$ $10^{10}$ radians/s, while the operating frequency is of the order $\omega \sim 3 \times 10^{12}$ radians/s. We therefore make the approximation $\omega_{e}^{\prime 2} /\left[k^{2} c^{2}-\omega^{2}\right]=\beta^{2} \gamma \omega_{e}^{2} / \omega^{2} \sim 10^{-5} \ll 1$, and simplify (13) as

$$
\left[\nabla_{t}^{2}-(k+r K)^{2}+\frac{\omega^{2}}{c^{2}}\right] E_{r}^{(e)}(x, y)=0 .
$$

As before, we Fourier transform the wave equation in $y$ and get

$$
\begin{aligned}
& {\left[\frac{\partial^{2}}{\partial x^{2}}-(k+r K)^{2}+\frac{\omega^{2}}{c^{2}}\right] \tilde{E}_{r}^{(e)}\left(x, k_{y}\right)} \\
& \quad+\frac{1}{\sqrt{2 \pi}} \int_{-\infty}^{\infty} d y e^{i k_{y} y} \frac{\partial^{2}}{\partial y^{2}} E_{r}^{(e)}(x, y)=0 .
\end{aligned}
$$

If we integrate by parts and ignore the discontinuities in $\partial E_{r}^{(e)} / \partial y$, for a broad optical beam with $\Delta x / \Delta y \ll 1$ [42], (15) becomes

$$
\frac{\partial^{2}}{\partial x^{2}} \tilde{E}_{r}^{(e)}\left(x, k_{y}\right)=\left[(k+r K)^{2}+k_{y}^{2}-\frac{\omega^{2}}{c^{2}}\right] \tilde{E}_{r}^{(e)}\left(x, k_{y}\right) .
$$

The solution is

$$
\tilde{E}_{r}^{(e)}\left(x, k_{y}\right)=\overline{\tilde{E}}_{r}^{(e)}\left(k_{y}\right) e^{-\alpha_{r}\left(k_{y}\right) x},
$$

where

$$
\alpha_{r}^{2}=(k+r K)^{2}+k_{y}^{2}-\frac{\omega^{2}}{c^{2}} .
$$

For real, positive $\alpha_{r}$ the wave amplitude vanishes in the limit $x \rightarrow \infty$, which corresponds to evanescent waves. Imaginary values of $\alpha_{r}$ correspond to waves that propagate away from the grating. For these waves, negative orders are synchronous with the electron velocity and form SmithPurcell radiation. Although these waves are excited by the electrons [25], they are not amplified and are much weaker than the evanescent waves. Propagating waves are also produced when the evanescent waves reach the ends of the grating. However, they are not synchronous with the electrons and they interact only weakly with the beam.
As in the grooves, the Maxwell equations relate $H_{r}^{(e) \prime}(x, y)$ and $E_{r}^{(e)}(x, y)$ above the grating by

$$
H_{r}^{(e) \prime}(x, y)=\frac{i \omega_{r}^{\prime} \varepsilon_{0}\left(1+\chi_{r}^{\prime}\right) c^{2}}{\omega^{2}-(k+r K)^{2} c^{2}} \frac{\partial}{\partial x} E_{r}^{(e)}(x, y),
$$

where $\chi_{r}^{\prime}=\chi_{e}^{\prime}\left(\omega_{r}^{\prime}\right)=-\omega_{e}^{\prime 2} / \omega_{r}^{\prime 2}$ and we have ignored $\omega_{e}^{\prime 2} \ll\left(k^{\prime}+r K^{\prime}\right)^{2} c^{2}$ in the denominator of the righthand side. To transform $H_{r}^{(e) \prime}(x, y)$ to the laboratory frame we use the Lorentz transformation

$$
H_{r}=\gamma\left(H_{r}^{\prime}+\beta c D_{r}^{\prime}\right)
$$

where $D_{r}^{\prime}$ is the $x$ component of the displacement field. From the Maxwell-Ampere law we have $\omega_{r}^{\prime} D_{r}^{\prime}=$ $\left(k^{\prime}+r K^{\prime}\right) H_{r}^{\prime}$. Combining this with (20), and recognizing that $\gamma\left[\omega_{r}^{\prime}+\beta\left(k^{\prime}+r K^{\prime}\right)\right]$ is the Lorentz transformation of $\omega$, we see that $H_{r}^{(e) \prime}(x, y)$ and $H_{r}^{(e)}(x, y)$ are related by $H_{r}^{(e)}(x, y)=\frac{\omega}{\omega_{r}^{\prime}} H_{r}^{(e) \prime}(x, y)$. The field in the laboratory frame is then

$$
H_{r}^{(e)}(x, y)=\frac{i \omega \varepsilon_{0}\left(1+\chi_{r}^{\prime}\right) c^{2}}{\omega^{2}-(k+r K)^{2} c^{2}} \frac{\partial}{\partial x} E_{r}^{(e)}(x, y) .
$$

Fourier transforming (21) in $y$, we get

$$
\begin{aligned}
\tilde{H}_{r}^{(e)}\left(x, k_{y}\right)= & \frac{i \omega \varepsilon_{0} c^{2}}{\omega^{2}-(k+r K)^{2} c^{2}}\left[\frac{\partial}{\partial x} \tilde{E}_{r}^{(e)}\left(x, k_{y}\right)\right. \\
& \left.+\frac{1}{\sqrt{2 \pi}} \int_{-\infty}^{\infty} d y e^{i k_{y} y} \chi_{r}^{\prime}(y) \frac{\partial}{\partial x} E_{r}^{(e)}(x, y)\right] .
\end{aligned}
$$

We simplify (22) by using the Faltung theorem to write the integral as

$$
\begin{aligned}
& \frac{1}{\sqrt{2 \pi}} \int_{-\infty}^{\infty} d y e^{i k_{y} y} \chi_{r}^{\prime}(y) \frac{\partial}{\partial x} E_{r}^{(e)}(x, y) \\
& =-\frac{1}{\sqrt{2 \pi}} \int_{-\infty}^{\infty} d k_{y}^{\prime} \tilde{\chi}_{r}^{\prime}\left(k_{y}-k_{y}^{\prime}\right) \alpha_{r}\left(k_{y}^{\prime}\right) \overline{\tilde{E}}_{r}^{(e)}\left(k_{y}^{\prime}\right) e^{-\alpha_{r}\left(k_{y}^{\prime}\right) x},
\end{aligned}
$$

where $k_{y}^{\prime}$ is a dummy variable and the Fourier transform of the susceptibility is

$$
\begin{aligned}
\tilde{\chi}_{e}^{\prime}\left(k_{y}-k_{y}^{\prime}\right) & =-\frac{1}{\sqrt{2 \pi}} \frac{\omega_{e}^{\prime 2}}{\omega_{r}^{\prime 2}} \int_{-W / 2}^{W / 2} d y e^{i\left(k_{y}-k_{y}^{\prime}\right) y} \\
& =-\frac{W}{\sqrt{2 \pi}} \frac{\omega_{e}^{\prime 2}}{\omega_{r}^{\prime 2}} \operatorname{sinc}\left[\frac{W}{2}\left(k_{y}-k_{y}^{\prime}\right)\right] .
\end{aligned}
$$

When written in terms of lab-frame variables, $\omega_{e}^{\prime 2} / \omega_{r}^{\prime 2}$ becomes

$$
\frac{\omega_{e}^{\prime 2}}{\omega_{r}^{\prime 2}}=\frac{\omega_{e}^{2}}{\gamma^{3}[\omega-\beta c(k+r K)]^{2}} .
$$

This is divergent near the synchronous point $\omega=\beta c k$ only for $r=0$. For all other $r \neq 0$ we may ignore the perturbation and rewrite (22) as 


$$
\begin{aligned}
\tilde{H}_{r}^{(e)}\left(x, k_{y}\right)= & \frac{i \omega \varepsilon_{0} c^{2}}{\omega^{2}-(k+r K)^{2} c^{2}}\left\{-\alpha_{r}\left(k_{y}\right) e^{-\alpha_{r}\left(k_{y}\right) x} \overline{\tilde{E}}_{r}^{(e)}\right. \\
& +\delta_{r 0} \frac{W}{2 \pi} \frac{\omega_{e}^{2}}{\gamma^{3}(\omega-\beta c k)^{2}} \int_{-\infty}^{\infty} d k_{y}^{\prime} \\
& \left.\times \operatorname{sinc}\left[\frac{W}{2}\left(k_{y}-k_{y}^{\prime}\right)\right] \alpha_{0}\left(k_{y}^{\prime}\right) \overline{\tilde{E}}_{0}^{(e)}\left(k_{y}^{\prime}\right) e^{-\alpha_{0}\left(k_{y}^{\prime}\right) x}\right\},
\end{aligned}
$$

where $\delta_{r 0}$ is the Kronecker delta.

Next we force continuity in $\tilde{E}_{z}$ and $\tilde{H}_{y}$ at the $x=0$ interface. In the grooves $(0<z<A)$, suppressing the harmonic time dependence, we have

$$
\begin{aligned}
& \tilde{E}_{z}^{(g)}\left(x=0, k_{y}\right)=\sum_{n=0}^{\infty} \overline{\tilde{E}}_{n}^{(g)}\left(k_{y}\right) \sinh \left[\kappa_{n}\left(k_{y}\right) H\right] \cos \left(\frac{n \pi}{A} z\right), \\
& \tilde{H}_{y}^{(g)}\left(x=0, k_{y}\right)=\sum_{n=0}^{\infty} \overline{\tilde{H}}_{n}^{(g)}\left(k_{y}\right) \cosh \left[\kappa_{n}\left(k_{y}\right) H\right] \cos \left(\frac{n \pi}{A} z\right),
\end{aligned}
$$

and on the teeth $(A<z<L)$

$$
\tilde{E}_{z}^{(g)}\left(x=0, k_{y}\right)=0 .
$$

Just above the grating, $\tilde{E}_{z}^{(e)}$ is given by

$$
\tilde{E}_{z}^{(e)}\left(x=0, k_{y}\right)=\sum_{r=-\infty}^{\infty} \overline{\tilde{E}}_{r}^{(e)}\left(k_{y}\right) e^{i(k+r K) z} .
$$

Setting (30) equal to, (27) and (29) multiplying both sides by $e^{-i(k+q K) z}$, and integrating over the grating period $L$, we get

$$
\overline{\tilde{E}}_{q}^{(e)}\left(k_{y}\right)=\frac{1}{L} \sum_{n=0}^{\infty} \overline{\tilde{E}}_{n}^{(g)}\left(k_{y}\right) \sinh \left[\kappa_{n}\left(k_{y}\right) H\right] K_{q n},
$$

where

$$
K_{q n}=i A \frac{(k+q K) A}{(k+q K)^{2} A^{2}-n^{2} \pi^{2}}\left[(-1)^{n} e^{-i(k+q K) A}-1\right] .
$$

Similarly, making $\tilde{H}_{y}$ continuous across the $x=0$ interface within the groove, multiplying both sides by $\cos \left(\frac{m \pi}{A} z\right)$, and integrating from 0 to $A$, we find

$$
\begin{gathered}
\overline{\tilde{H}}_{m}^{(g)}\left(k_{y}\right) \frac{1+\delta_{m 0}}{2} A \cosh \left[\kappa_{m}\left(k_{y}\right) H\right] \\
=\sum_{r=-\infty}^{\infty} \tilde{H}_{r}^{(e)}\left(0, k_{y}\right) K_{r m}{ }^{*} .
\end{gathered}
$$

We may now substitute expressions (9) and (26) into (33), remembering to evaluate at $x=0$, and then use (31) to arrive at the dispersion relation

$$
\overline{\tilde{E}}_{m}^{(g)}\left(k_{y}\right)=\sum_{n=0}^{\infty}\left\{R_{m n}\left(k_{y}\right) \overline{\tilde{E}}_{n}^{(g)}\left(k_{y}\right)+S_{m n}\left[k_{y}, \overline{\tilde{E}}_{n}^{(g)}\left(k_{y}\right)\right]\right\} .
$$

The first term in the square brackets represents modes admitted by the empty (no beam) grating. The second term embodies the modification of those modes by the presence of the electron beam. The matrix elements are given by

$$
\begin{aligned}
R_{m n}\left(k_{y}\right)= & -\frac{4}{1+\delta_{m 0}} \frac{A}{L} \frac{\sinh \left[\kappa_{n}\left(k_{y}\right) H\right]}{\cosh \left[\kappa_{m}\left(k_{y}\right) H\right]} \frac{\omega^{2}-\frac{m^{2} \pi^{2}}{A^{2}} c^{2}}{\kappa_{m}\left(k_{y}\right)} \sum_{r=-\infty}^{\infty} \frac{(k+r K) A}{(k+r K)^{2} A^{2}-n^{2} \pi^{2}} \frac{(k+r K) A}{(k+r K)^{2} A^{2}-m^{2} \pi^{2}} \\
& \times \frac{\alpha_{r}\left(k_{y}\right)}{\omega^{2}-(k+r K)^{2} c^{2}} \begin{cases}1-(-1)^{m} \cos [(k+r K) A] & m+n=\text { even } \\
i(-1)^{m} \sin [(k+r K) A] & m+n=\text { odd }\end{cases}
\end{aligned}
$$

and

$$
\begin{aligned}
S_{m n}\left(k_{y}, \overline{\tilde{E}}_{n}^{(g)}\right)= & \frac{W}{A L \pi\left(1+\delta_{m 0}\right)} \frac{1}{\cosh \left[\kappa_{m}\left(k_{y}\right) H\right]} \frac{\omega^{2}-\frac{m^{2} \pi^{2}}{A^{2}} c^{2}}{\kappa_{m}\left(k_{y}\right)} \frac{K_{0 n} K_{0 m}{ }^{*}}{\omega^{2}-k^{2} c^{2}} \frac{\omega_{e}^{2}}{\gamma^{3}[\omega-\beta c k]^{2}} \int_{-\infty}^{\infty} d k_{y}^{\prime} \operatorname{sinc}\left[\frac{W}{2}\left(k_{y}-k_{y}^{\prime}\right)\right] \\
& \times \alpha_{0}\left(k_{y}^{\prime}\right) \sinh \left[\kappa_{n}\left(k_{y}^{\prime}\right) H\right] \overline{\tilde{E}}_{n}^{(g)}\left(k_{y}^{\prime}\right) .
\end{aligned}
$$

Calculations show that the dispersion relation for the grating is well described using a single term $(m, n=0)$ for the groove fields, provided that we use at least three space harmonics $(-1<r<1)$ to describe the fields above the grating. We therefore define the dispersion function $D\left(\omega, k, k_{y}\right)$ as

$$
D\left(\omega, k, k_{y}\right)=1-R_{00}\left(\omega, k, k_{y}\right) .
$$

The dispersion curve $D(\omega, k, 0)=0$, for waves traveling normal to the grooves of the empty grating described by Table I, is plotted in Fig. 3, along with 30-kV and $150-\mathrm{kV}$ beam lines. At the intersection of these curves the phase velocity of the evanescent wave and the electron beam are synchronous, i.e. $\omega / k=\beta c$. Energy in the evanescent mode will travel along the grating at the group velocity $\beta_{g}=d \omega / d k$, which is negative in the right half of the 
Brillouin zone. When the evanescent wave reaches the end of the grating, it scatters into free space. Some of the radiation is reflected into an evanescent wave traveling in the opposite direction, but the reflection coefficient is found to be small, typically on the order of $10 \%$ [32].

When the electron beam is present, the dispersion relation (34) for $m=n=0$ becomes

$$
\begin{aligned}
D\left(\omega, k, k_{y}\right) \overline{\tilde{E}}_{0}^{(g)}\left(k_{y}\right)= & \frac{W}{\pi A L k^{2}} \frac{\omega_{e}^{2}}{\gamma^{3}[\omega-\beta c k]^{2}} \frac{1-\cos (k A)}{\cosh \left[\kappa_{0}\left(k_{y}\right) H\right]} \frac{\omega^{2}}{\kappa_{0}\left(k_{y}\right)} \frac{1}{\omega^{2}-k^{2} c^{2}} \int_{-\infty}^{\infty} d k_{y}^{\prime} \operatorname{sinc}\left[\frac{W}{2}\left(k_{y}-k_{y}^{\prime}\right)\right] \alpha_{0}\left(k_{y}^{\prime}\right) \\
& \times \sinh \left[\kappa_{0}\left(k_{y}^{\prime}\right) H\right] \overline{\tilde{E}}_{0}^{(g)}\left(k_{y}^{\prime}\right) .
\end{aligned}
$$

We expect that the gain will be maximal near the synchronous point $\left(\omega, k, k_{y}\right)=\left(\omega_{0}, k_{0}, 0\right)$, where

$$
\begin{gathered}
D\left(\omega_{0}, k_{0}, 0\right)=0, \\
\omega_{0}=\beta c k_{0} .
\end{gathered}
$$

Near the synchronous point, we may expand the dispersion function in a Taylor series about $\left(\omega_{0}, k_{0}, 0\right)$. Since $k_{y}$ appears in the dispersion function only as $k_{y}^{2}$, we expand as

$$
D\left(\omega, k, k_{y}\right) \approx D_{\omega} \delta \omega+D_{k} \delta k+D_{y} k_{y}^{2},
$$

where

$$
\begin{gathered}
\delta \omega=\omega-\omega_{0}, \\
\delta k=k-k_{0},
\end{gathered}
$$

and

$$
\begin{aligned}
& D_{\omega}\left(\omega_{0}, k_{0}\right)=\frac{\partial D}{\partial \omega}\left(\omega_{0}, k_{0}, 0\right), \\
& D_{k}\left(\omega_{0}, k_{0}\right)=\frac{\partial D}{\partial k}\left(\omega_{0}, k_{0}, 0\right), \\
& D_{y}\left(\omega_{0}, k_{0}\right)=\frac{\partial D}{\partial k_{y}^{2}}\left(\omega_{0}, k_{0}, 0\right) .
\end{aligned}
$$

Since the dispersion function vanishes everywhere along

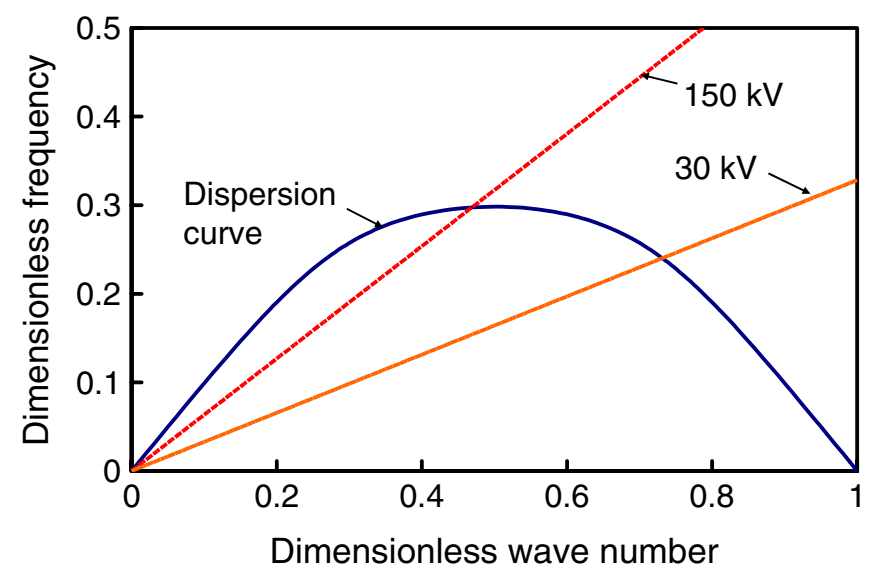

FIG. 3. (Color) Dimensionless grating dispersion curve $(\omega / c K$ versus $k / K)$ and electron beam lines for 30 and $150 \mathrm{kV}$. the dispersion curve, the derivatives $D_{\omega}$ and $D_{k}$ are related by

$$
\frac{d D}{d k}=0=\frac{\partial D}{\partial k}+\frac{\partial D}{\partial \omega} \frac{\partial \omega}{\partial k}=D_{k}+\beta_{g} c D_{\omega},
$$

where $\beta_{g} c=\partial \omega / \partial k$ is the group velocity. This allows us to rewrite the dispersion function as

$$
D\left(\omega, k, k_{y}\right) \cong D_{\omega}\left(\delta \omega-\beta_{g} c \delta k\right)+D_{y} k_{y}^{2} .
$$

For modes that are broad compared with the wavelength, $k_{y} \ll k \approx O(\omega / c)$, so we can make the approximations

$$
\alpha_{0}\left(\omega, k, k_{y}\right) \approx \alpha_{0}\left(\omega_{0}, k_{0}, 0\right)=\frac{\omega_{0}}{\beta \gamma c},
$$

and

$$
\kappa_{0}\left(\omega, k, k_{y}\right) \approx \kappa_{0}\left(\omega_{0}, k_{0}, 0\right)=i \frac{\omega_{0}}{c},
$$

near the synchronous point. In other words, the vertical profile of a broad evanescent wave is close to that of a $2 \mathrm{D}$ wave. We also note that

$$
\omega-\beta c k=\delta \omega-\beta c \delta k
$$

Including these approximations, the dispersion (38) relation becomes

$$
\begin{gathered}
(\delta \omega-\beta c \delta k)^{2}\left[D_{\omega}\left(\delta \omega-\beta_{g} c \delta k\right)+D_{y} k_{y}^{2}\right] \overline{\tilde{E}}_{0}^{(g)}\left(k_{y}\right) \\
=\Delta \frac{W D_{\omega}}{2 \pi} \int_{-\infty}^{\infty} d k_{y}^{\prime} \operatorname{sinc}\left[\frac{W}{2}\left(k_{y}-k_{y}^{\prime}\right)\right] \overline{\tilde{E}}_{0}^{(g)}\left(k_{y}^{\prime}\right),
\end{gathered}
$$

where

$$
\Delta=-\frac{2 \beta^{3} c^{2}}{A L D_{\omega}} \frac{\omega_{e}^{2}}{\gamma^{2} \omega_{0}^{2}} \tan \left(\frac{\omega_{0}}{c} H\right)\left[1-\cos \left(k_{0} A\right)\right],
$$

Computations show that $D_{\omega}$ is real negative, so $\Delta$ is real positive.

This result may be compared to the 2D theory of the grating FEL by taking the limit as $W \rightarrow \infty$ and subsequently evaluating at $k_{y}=0$. In this limit, the sinc function behaves as a delta function and selects out the integrand value at $k_{y}^{\prime}=k_{y}=0$. Evaluating (52) gives the cubic dispersion relation

$$
(\delta \omega-\beta c \delta k)^{2}\left(\delta \omega-\beta_{g} c \delta k\right)=\Delta .
$$


This is the dispersion relation obtained previously for the 2D theory [31]. It agrees exactly with the results obtained by Mkrtchian [34], who begins with the electron equations of motion and the reflection matrix for the grating. This confirms the equivalence of the plasma susceptibility used in the present approach. Results computed using (54) are also confirmed by PIC simulations [32].

Of greater interest is the limit in which the electron beam is very narrow compared to the mode width. In this case, $\operatorname{sinc}\left[\frac{W}{2}\left(k_{y}-k_{y}^{\prime}\right)\right] \approx 1$, and the remaining integral is recognized as $\sqrt{2 \pi} \bar{E}_{0}^{(g)}(0)$. The dispersion relation is simplified by the substitution

$$
\Delta k_{y}^{2}=-\frac{D_{\omega}}{D_{y}}\left(\delta \omega-\beta_{g} c \delta k\right) .
$$

The roots of this equation are $\Delta k_{y}^{(+)}$and $\Delta k_{y}^{(-)}=-\Delta k_{y}^{(+)}$, which lie above and below the real axis, respectively. Solving (52) for $\overline{\tilde{E}}_{0}^{(g)}$ and inverting the Fourier transform, we get

$$
\frac{(\delta \omega-\beta c \delta k)^{2}}{W \Delta} \frac{D_{y}}{D_{\omega}} \bar{E}_{0}^{(g)}(y)=\frac{\bar{E}_{0}^{(g)}(0)}{2 \pi} \int_{-\infty}^{\infty} d k_{y} \frac{e^{-i k_{y} y}}{k_{y}^{2}-\Delta k_{y}^{2}} .
$$

The integrand has poles at $\Delta k_{y}^{(+)}$and $\Delta k_{y}^{(-)}=-\Delta k_{y}^{(+)}$. The integral is evaluated using contour integration and the residue theorem. For $y>0$ the contour is closed in the lower half plane, enclosing the pole at $\Delta k_{y}^{(-)}$, so the integrand vanishes along the curved segment. Integrating clockwise around the contour, we find

$$
\bar{E}_{0}^{(g)}(y)=-\frac{i}{2} W \Delta \frac{D_{\omega}}{D_{y}} \frac{\bar{E}_{0}^{(g)}(0)}{(\delta \omega-\beta c \delta k)^{2}} \frac{e^{-i \Delta k_{y}^{(-)} y}}{\Delta k_{y}^{(-)}} .
$$

Since $\operatorname{Im}\left(\Delta k_{y}^{(-)}\right)<0$, the field vanishes at $y=\infty$, as required. Similarly, for $y<0$ the contour is closed in the upper half plane, enclosing the pole at $\Delta k_{y}^{(+)}$. Since $\operatorname{Im}\left(\Delta k_{y}^{(+)}\right)>0$, the field vanishes at $y=-\infty$. Evaluating (57) at $y=0$ and rearranging, we get

$$
(\delta \omega-\beta c \delta k)^{2} \Delta k_{y}^{(-)}=-\frac{i}{2} W \Delta \frac{D_{\omega}}{D_{y}} .
$$

Squaring this result and using (55), we arrive at the dispersion relation for the narrow beam case,

$$
(\delta \omega-\beta c \delta k)^{4}\left(\delta \omega-\beta_{g} c \delta k\right)=\frac{1}{4} W^{2} \Delta^{2} \frac{D_{\omega}}{D_{y}} .
$$

This fifth-degree equation admits five roots, but not all are physically allowed. Rearranging (58), we see that

$$
(\delta \omega-\beta c \delta k)^{2}=-\frac{i}{2} \frac{W \Delta}{\Delta k_{y}^{(-)} \Delta k_{y}^{(-) *}} \frac{D_{\omega}}{D_{y}} \Delta k_{y}^{(-) *},
$$

where $\Delta k_{y}^{(-) *}$ lies above the real axis. Calculations show

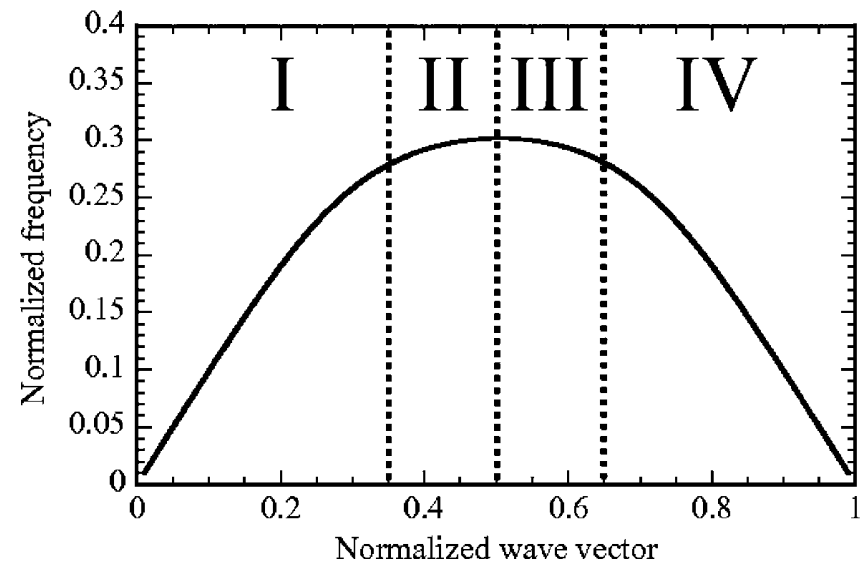

FIG. 4. Division of operating regions by transverse diffraction.

that $D_{\omega}$ is negative, irrespective of $k$, so only those roots are physically allowed (the fields vanish at infinity) for which

$$
\operatorname{Re}\left[D_{y}\left(\delta \omega-\beta_{g} c \delta k\right)^{2}\right]<0 .
$$

But $D_{y}$ changes sign such that $D_{y}>0$ near the center of the Brillouin zone $(k / K=1 / 2)$ and $D_{y}<0$ towards the edges of the zone $(k=0, K)$. This subdivides the Brillouin zone into the four distinct regions pictured in Fig. 4. We now consider separately the amplifier and oscillator regimes of the grating FEL.

\section{AMPLIFIER}

In the left half of the Brillouin zone, the group velocity is positive and the device operates on a convective instability, as an amplifier. When the device operates as a steady-state amplifier, the frequency is fixed by the input. Since the gain is largest at the synchronous condition [31], we may take $\delta \omega=0$, and $\beta_{g}$ is positive. The dispersion relation (59) becomes

$$
\delta k^{5}=\Gamma,
$$

where

$$
\Gamma=-\frac{W^{2} \Delta^{2}}{4 \beta^{4} \beta_{g} c^{5}} \frac{D_{\omega}}{D_{y}} .
$$

In region I, where $D_{y}<0$ and $\Gamma<0$, the roots are given by

$$
\delta k_{n}=|\Gamma|^{1 / 5} e^{i(2 n+1) \pi / 5} .
$$

These are shown in Fig. 5. In region I there are three physically allowed roots $(n=0,2,4)$. The roots for $n=$ 0,4 correspond to slow waves, but $n=2$ corresponds to a fast wave. It is surprising that a fast wave is allowed in a gain-guided system, since we are familiar with optical fibers that depend on an index of refraction greater than unity. The root corresponding to $n=4$ has an amplitude 


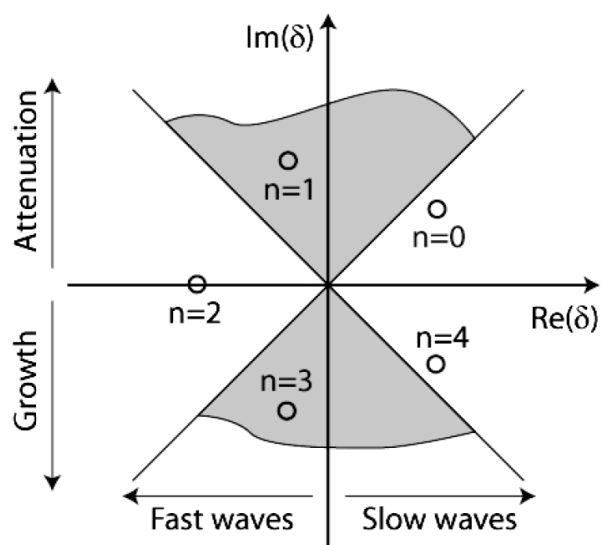

FIG. 5. Roots of the dispersion relation for region I. Forbidden regions are shaded.

growth rate (half the intensity gain coefficient) $\mu=$ $-\operatorname{Im} \delta k$ given by

$$
\mu=|\Gamma|^{1 / 5} \sin (\pi / 5) .
$$

Similarly, in region II, where $D_{y}>0$ and $\Gamma>0$, the roots of (62) are

$$
\delta k_{n}=|\Gamma|^{1 / 5} e^{i 2 n \pi / 5},
$$

for $n=0, \ldots, 4$. These are plotted in the complex plane in Fig. 6. From (61) we find that only the two roots corresponding to $n=1,4$ are physically allowed. From Fig. 6 we see that both of these are slow waves $(\operatorname{Re} \delta k>0)$, i.e., they have a phase velocity lower than that of the synchronous point. The amplitude growth rate for the root $n=4$ is

$$
\mu=|\Gamma|^{1 / 5} \sin (2 \pi / 5) .
$$

The transverse decay rate of the field is found from (55) to be

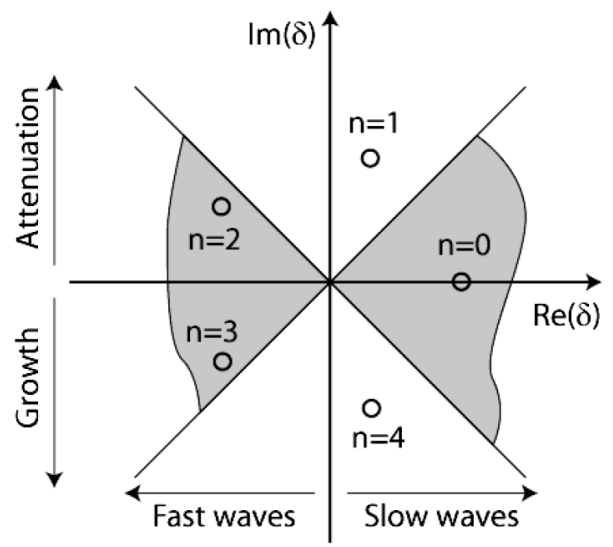

FIG. 6. Roots of the dispersion relation for region II. Forbidden regions are shaded.

$$
\begin{aligned}
\Delta k_{y}^{2} & =-\left|\frac{D_{\omega}}{D_{y}} \beta_{g} c \Gamma^{1 / 5}\right| e^{i 2 n \pi / 5} \\
& =\left|\frac{D_{\omega}}{D_{y}} \beta_{g} c \Gamma^{1 / 5}\right| e^{i(2 n-5) \pi / 5},
\end{aligned}
$$

so the corresponding $1 / e$ width of the mode $n=4$ is

$$
\Delta y=-\frac{2}{\operatorname{Im}\left(\Delta k_{y}\right)}=\frac{2}{\sin (3 \pi / 10)}\left|\frac{D_{\omega}}{D_{y}} \beta_{g} c \Gamma^{1 / 5}\right|^{-1 / 2} .
$$

As an example, we consider the grating and beam described in Table I. For this particular grating the Bragg point $\left(\beta_{g}=0\right)$ is located at $V_{\text {Bragg }} \approx 126 \mathrm{kV}$. An operating voltage of $150 \mathrm{kV}$ places the operating point in region II. To compensate for errors introduced by our assumption that the beam stretches from the grating top to infinity, we scale the electron density by the filling factor [34]

$$
F_{\text {fill }}=e^{-\left[b_{e} /(\Delta x)\right]}-e^{-\left[t_{e} /(\Delta x)\right]},
$$

where $b_{e}$ is the bottom of the electron beam and $t_{e}$ the top of the beam relative to the grating surface, and $\Delta x=$ $1 / 2 \alpha_{0}(0)=\beta \gamma \lambda / 4 \pi$ is the intensity scale height for the synchronous evanescent wave. For this case the filling factor is $F_{\text {fill }} \approx 0.8$. The three-dimensional growth rate is plotted in Fig. 7, along with the two-dimensional value. When diffraction is included, the growth rate is reduced by about a factor of 3 . The $1 / e$ amplitude mode width is found to be $\Delta y \approx 5.5 \mathrm{~mm}$. This is much wider than the electron beam, the grating period, and the scale height of the evanescent wave. Thus, it is clear that the initial assumptions made concerning its dimensions are justified. In the vicinity of $326 \mathrm{kV}$, the growth rate diverges, as shown in Fig. 7. This is the boundary between region I and region II, and the mode width vanishes here. This violates the assumption that the mode is wide, so the theory is not strictly applicable in this neighborhood.

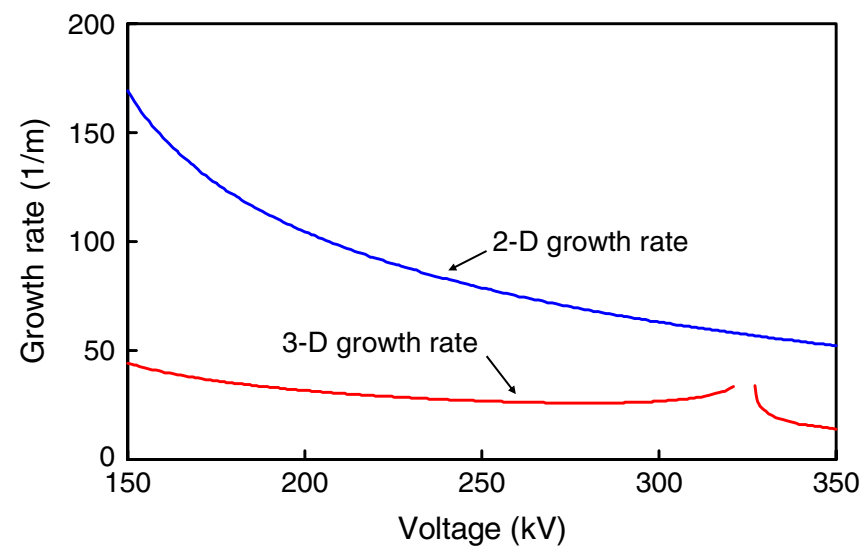

FIG. 7. (Color) Growth rate for the parameters of Table I. 


\section{OSCILLATOR}

When the synchronous point is to the right of the Bragg point, the group velocity of the evanescent mode is negative. This allows energy exchanged in the beam-wave interaction to be transported to the upstream end of the grating. This serves as an intrinsic form of feedback, called an absolute instability. Provided that the beam current exceeds the so-called start current, the device oscillates spontaneously. In this case, both the frequency shift $\delta \omega$ and the wave number shift $\delta k$ are complex. The waves corresponding to the several roots of the dispersion relation become locked together to form the mode of the oscillator. All the waves have the same (complex) frequency shift $\delta \omega_{j}=\delta \omega$, but different wave number shifts. This allows them to interfere constructively and destructively to satisfy the boundary conditions at the ends of the grating $[47,48]$.

To estimate the start current for oscillation, we express the electric field above the grating as a sum of the fields of the modes corresponding to the physically allowed roots of the dispersion relation (59). The field of the $j$ th mode is

$$
E_{j}=\sum_{p=-\infty}^{\infty} E_{p}^{(j)} e^{-\alpha_{p}^{(j)} x} e^{-i \Delta k_{y}^{(j)} y} e^{i p K z} e^{i\left(k_{0} z-\omega_{0} t\right)} e^{i\left(\delta k_{j} z-\delta \omega t\right)} .
$$

To lowest order in the perturbation, however, the coefficients $E_{p}^{(j)}$ and $\alpha_{p}^{(j)}$ are the same as for the empty structure and therefore independent of $j$, so the field above the grating at any time may be expressed

$$
E_{x}=\sum_{j} A_{j} E_{j}=E_{0} \sum_{j} A_{j} e^{i\left(\Delta k_{y}^{(j)} y+\delta k_{j} z-\delta \omega t\right)},
$$

where the coefficients $A_{j}$ are constants and

$$
E_{0}=\sum_{p=-\infty}^{\infty} E_{p} e^{-\alpha_{p} x} e^{i p K z} e^{i\left(k_{0} z-\omega_{0} t\right)}
$$

At the upstream end of the grating, the plasma enters undisturbed in density and velocity. Since the density fluctuations vanish, the polarization of the beam vanishes, and since the velocity fluctuations vanish, the convective derivative of the polarization vanishes. But from (12) and (25) we find that the polarization is

$$
P_{x}=-\frac{\varepsilon_{0} \omega_{p}^{2} E_{0}}{\gamma^{3}} \sum_{j} \frac{A_{j} e^{i\left(\delta k_{j} x-\delta \omega t\right)}}{\left(\delta \omega-\beta c \delta k_{j}\right)^{2}}
$$

where we have taken $y=0$ since the electron beam is narrowly confined to the region near the axis. The convective derivative of the polarization is

$$
\frac{d P_{x}}{d t}=\left(\frac{\partial}{\partial t}+\beta c \frac{\partial}{\partial x}\right) P_{x}=\frac{i \varepsilon_{0} \omega_{p}^{2} E_{0}}{\gamma^{3}} \sum_{j} \frac{A_{j} e^{i\left(\delta k_{j} x-\delta \omega t\right)}}{\delta \omega-\beta c \delta k_{j}} .
$$

The boundary conditions therefore become

$$
\begin{gathered}
\sum_{j} \frac{A_{j}}{\left(\delta \omega-\beta c \delta k_{j}\right)^{2}}=0, \\
\sum_{j} \frac{A_{j}}{\delta \omega-\beta c \delta k_{j}}=0 .
\end{gathered}
$$

For the third boundary condition, we assume that there is no input field at the downstream end of the grating. If we ignore reflections at the ends of the grating [32], the field at the right end of the grating vanishes. For a grating of length $Z$, the corresponding boundary condition is

$$
\sum_{j} A_{j} e^{i\left(\Delta k_{y}^{(j)} y+\delta k_{j} Z\right)}=0 .
$$

This must be satisfied for all $y$. However, the transverse decay rates $\Delta k_{y}^{(j)}$ are not all the same, so the three modes themselves cannot satisfy the boundary condition everywhere. We presume that away from the axis, the differences are made up by adding in waves that are not synchronous nor guided by the beam. Since they are not synchronous and do not propagate up the grating, these waves have negligible interaction with the beam and can be ignored. We therefore apply the boundary condition at (78) $y=0$. Since the optical-mode experiences many e-folds as it propagates to the upstream end of the grating, the exponential coefficients that we seek are only logarithmically influenced by the details of the coefficients at the downstream end of the grating.

The boundary conditions (76)-(78) must be solved subject to the constraint imposed by the dispersion relation (59). For convenience, we introduce the dimensionless variables,

$$
\begin{gathered}
\delta_{j}=-\hat{D}_{y}\left|\frac{4}{W^{2} \Delta^{2}} \frac{D_{y}}{D_{\omega}} \frac{\beta_{g}}{\beta}\right|^{1 / 5}\left(\delta \omega-\beta c \delta k_{j}\right), \\
\zeta=-\frac{\hat{D}_{y} Z}{\beta c}\left|\frac{W^{2} \Delta^{2}}{4} \frac{D_{\omega}}{D_{y}} \frac{\beta}{\beta_{g}}\right|^{1 / 5},
\end{gathered}
$$

where $\hat{D}_{y}=\operatorname{sign}$ of $D_{y}$, and write the boundary conditions in the form

$$
\begin{gathered}
\sum_{j} \frac{A_{j}}{\delta_{j}^{2}}=0, \\
\sum_{j} \frac{A_{j}}{\delta_{j}}=0, \\
\sum_{j} A_{j} e^{-i \zeta \delta_{j}}=0 .
\end{gathered}
$$

For a solution to exist, it is necessary that the determinant of the coefficients vanish, 


$$
\left|\begin{array}{ccc}
1 / \delta_{1}^{2} & 1 / \delta_{2}^{2} & 1 / \delta_{3}^{2} \\
1 / \delta_{1} & 1 / \delta_{2} & 1 / \delta_{3} \\
e^{-i \zeta \delta_{1}} & e^{-i \zeta \delta_{2}} & e^{-i \zeta \delta_{3}}
\end{array}\right|=0 .
$$

Finally, we introduce the dimensionless frequency shift

$$
\nu=-\hat{D}_{y}\left|\frac{4}{W^{2} \Delta^{2}} \frac{D_{y}}{D_{\omega}} \frac{\beta_{g}}{\beta}\right|^{1 / 5}\left(\frac{\beta_{g}-\beta}{\beta_{g}} \delta \omega\right),
$$

in terms of which the dispersion relation becomes (for $\beta_{g}<0$ )

$$
\delta^{4}(\delta-\nu)+1=0 .
$$

The roots of this equation must satisfy the condition (61), which can now be expressed:

$$
\hat{D}_{y} \operatorname{Re}\left(\delta^{2}\right)<0 .
$$

At threshold, the growth rate vanishes, so $\operatorname{Im} \delta \omega=$ $\operatorname{Im} \nu=0$. Computations for real $\nu$ show that for three of the roots $\operatorname{Re}\left(\delta^{2}\right)>0$, while for two of the roots $\operatorname{Re}\left(\delta^{2}\right)<$ 0 . Thus, there are three physically allowed roots in region IV $\left(\hat{D}_{y}<0\right)$ and two in region III. We see from this that for a narrow electron beam over a wide grating, there are not enough physically allowed (gain-guided) modes in region III to satisfy the boundary conditions (84). Operation in this part of the Brillouin zone will require either a wide electron beam or a grating of finite width to confine the optical mode so that diffraction is not important and gain guiding is not required. In the following, we confine the discussion to region IV.

The dimensionless equations (84) and (86) must be solved numerically. It is found that the smallest value of $\zeta$ for which the imaginary part of $\nu$ is nonnegative is $\zeta_{0}=$ 1.1938. Thus, the threshold condition for a growing oscillation is

$$
\frac{Z}{\beta c}\left|\frac{W^{2} \Delta^{2}}{4} \frac{D_{\omega}}{D_{y}} \frac{\beta}{\beta_{g}}\right|^{1 / 5}-\zeta_{0}=0 .
$$

For the parameters of the Dartmouth experiment the pre-

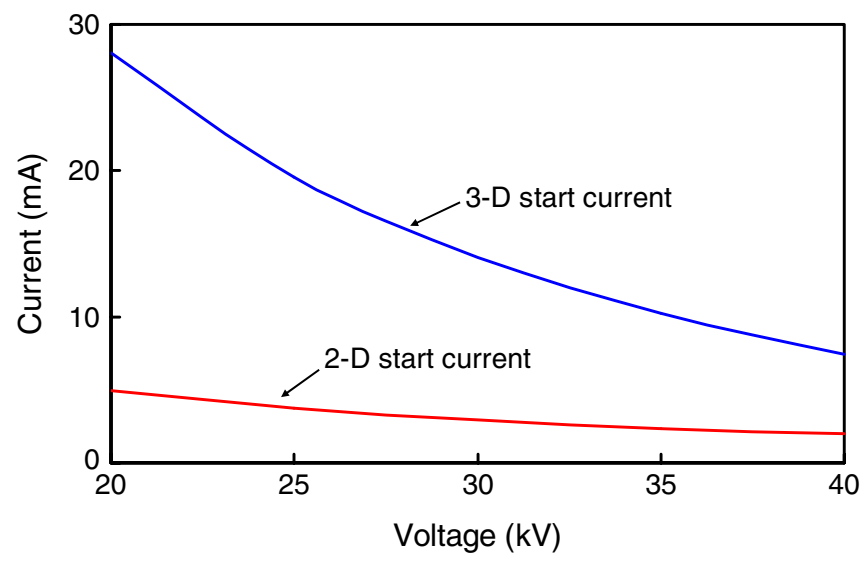

FIG. 8. (Color) Start current for the parameters of Table I. dicted start current is shown in Fig. 8. The start current predicted by the $3 \mathrm{D}$ theory is about 5 times higher than that predicted by the $2 \mathrm{D}$ theory [32].

\section{DISCUSSION}

We have presented a three-dimensional theory of a grating FEL operating as an amplifier in the exponential gain regime and as an oscillator. For a wide electron beam, the cubic dispersion relation obtained in previous analyses of slow-wave structures $[8,49]$ is recovered. However, when the electron beam is narrow, gain guiding results in a fifth-order dispersion relation. From (65) we see that the growth rate and the electron beam current in this case are related by

$$
\mu \propto n_{e}^{2 / 5} \propto I^{2 / 5},
$$

where $I$ is the electron beam total current. To understand this result we consider the relationship found in previous two-dimensional analyses [32-34], given by

$$
\mu \propto \bar{I}^{1 / 3},
$$

where $\bar{I}$ is the current density. In the three-dimensional case, the optical mode spreads by diffraction over a length on the order of the gain length $Z_{g}=1 / \mu$ to a width on the order of $\Delta y \propto \sqrt{Z_{g}}$. The effective average current density over the width of the mode is then

$$
\bar{I} \propto \frac{I}{\Delta y} \propto \frac{I}{\sqrt{Z_{g}}} .
$$

Combining this with (90), we get

$$
\mu \propto I^{2 / 5} .
$$

This relationship is simply understood to be the manifestation of gain guiding in the grating FEL. Transverse diffraction results in strong dilution of the amplifier gain. Similar effects are observed in the 3D theory of the Cerenkov FEL [42].

More surprising are the unexpected results of diffraction in a slow-wave structure in which the group velocity is very different from the phase velocity, and may be negative. Conventionally, optical guiding by a fiber with an index of refraction greater than unity is the result of interference between the phase fronts of the guided wave. However, when the phase velocity is negative, so that the optical pulse is traveling backwards, it is more difficult to understand the effects of interference of the phase fronts, which are moving to the right, on the guiding of a pulse that is moving to the left. We find for our periodic structure that in regions I and IV one of the three guided modes is a fast wave (for which the electron beam has an effective index of refraction less than unity), while in regions II and III there are only two guided modes.

Up to this time, it has been impossible to do numerical simulations of a grating FEL with a wide grating, due to 
limitations of computer memory, so there are no simulations to compare the theory to. Computations for a grating of finite width show that the edges of the grating have a strong influence on the modes above the grating $[36,44]$. The only published experimental results for a wide grating are those of the Dartmouth group [26]. The parameters of those experiments are summarized in Table I; the electron energy was $30 \mathrm{keV}$. The start current predicted for those experiments is shown in Fig. 8. In the Dartmouth experiments, nonlinear (superradiant) Smith-Purcell radiation was observed at currents above about $1 \mathrm{~mA}$. However, this is more than an order of magnitude below the predicted start current, so it is unlikely that the evanescent wave was oscillating. Moreover, radiation at the frequency of the evanescent wave was never observed. Since nonlinear emission was observed to turn on at about the same current $(\sim 1 \mathrm{~mA})$ in other devices using this same electron beam [28], including a Cerenkov laser [50], it is possible that the coherence was caused by modulation of the current by an electron beam instability. This conjecture is consistent with other evidence. In experiments having parameters very close to those of the Dartmouth group, but using a different electron gun, Kapp et al. [29] saw no nonlinear radiation, while in experiments using a modification of the electron gun used in the Dartmouth experiments, Andrews et al. [35] saw strongly nonlinear emission. They also saw radiation from the evanescent wave scattering off the ends of the grating. However, while the beam current used in those experiments was close to the predicted start current [38], there was no clear evidence of oscillation. This is likely due to Joule losses in the grating surface, which are important at $\mathrm{THz}$ frequencies [31], and to imperfections in the gratings.

\section{ACKNOWLEDGMENTS}

The authors gratefully acknowledge helpful discussions with K.-J. Kim and J.T. Donohue. This work was supported by the Medical Free Electron Laser Program of the Department of Defense under Grant No. F49620-01-10429.

[1] S. P. Mickan and X.-C. Zhang, Int. J. High Speed Electron. Sys. 13, 601 (2003).

[2] P. H. Siegel, IEEE Trans. Microwave Theory Tech. 50, 910 (2002).

[3] M. Abo-Bakr, J. Feikes, K. Holldack, G. Wuestefeld, and H.-W. Huebers, Phys. Rev. Lett. 88, 254801 (2002).

[4] G. P. Williams, Rev. Sci. Instrum. 73, 1461 (2002).

[5] G. Ramian, Nucl. Instrum. Methods Phys. Res., Sect. A 318, 225 (1992).

[6] H. Koike, M. Sobajima, V. M. Popik, M. Yokoyama, F. Oda, M. Kawai, K. Toyoda, H. Kuroda, and K. Nakai, Nucl. Instrum. Methods Phys. Res., Sect. A 507, 242 (2003).
[7] N. A. Vinokurov, V.P. Bolotin, D. A. Kayran, B. A. Knyazev, E. I. Kolobanov, V. V. Kotenkov, V. V. Kubarev, G. N. Kulipanov, A. N. Matveenko, L. E. Medvedev, S. V. Miginsky, L. A. Mironenko, A. D. Oreshkov, V. K. Ovchar, V. M. Popik, T. V. Salikova, M.A. Scheglov, S.S. Serednyakov, O.A. Shevchenko, and A. N. Skrinsky, International FEL Conference, Trieste, 2004, http:// fel2004.elettra.trieste.it/pls/fel2004/Proceedings.html.

[8] J.R. Pierce, Traveling Wave Tubes (Van Nostrand Company, New York, 1950).

[9] D. E. Wortman and R.P. Leavitt, in Infrared and Millimeter Waves, edited by K. J. Button (Academic, New York, 1979), Vol. 7.

[10] G. Kantorowicz and P. Palluel, in Infrared and Millimeter Waves, edited by K. J. Button (Academic, New York, 1979).

[11] Insight Product Company, http://www.insight-product. com/submmbwo3.htm.

[12] S. J. Smith and E. M. Purcell, Phys. Rev. 92, 1069 (1953).

[13] P. M. van den Berg, J. Opt. Soc. Am. 63, 689 (1973).

[14] P. M. van den Berg, J. Opt. Soc. Am. 63, 1588 (1973).

[15] P. M. van den Berg and T. H. Tan, J. Opt. Soc. Am. 64, 325 (1974).

[16] C. A. Brau, Modern Problems in Classical Electrodynamics (Oxford, New York, 2004), p. 532.

[17] D. Li, Z. Yang, K. Imasaki, and Gun-Sik Park, Phys. Rev. ST Accel. Beams 9, 040701 (2006).

[18] Y. Shibata, S. Hasebe, K. Ishi, S. Ono, M. Ikezawa, T. Nakazato, M. Oyamada, S. Urasawa, T. Takahashi, T. Matsuyama, K. Kobayashi, and Y. Fujita, Phys. Rev. E 57, 1061 (1998).

[19] A.S. Kesar, Phys. Rev. ST Accel. Beams 8, 072801 (2005).

[20] S. E. Korbly, A. S. Kesar, J. R. Sirigiri, and R. J. Temkin, Phys. Rev. Lett. 94, 054803 (2005).

[21] C. S. Liu and V. K. Tripathi, IEEE J. Quantum Electron. 35, 1386 (1999).

[22] B. K. Skrynnik, V. K. Korneenkov, and M. Y. Demchenko, Telecommun. Radio Eng. 55, 154 (2001).

[23] H. L. Andrews, C. H. Boulware, C. A. Brau, and J.D. Jarvis, Phys. Rev. ST Accel. Beams 8, 110702 (2005).

[24] J. T. Donohue and J. Gardelle, Phys. Rev. ST Accel. Beams 8, 060702 (2005).

[25] D. Li, K. Imasaki, Z. Yang, G.-S. Park, S. Miyamoto, S. Amano, and T. Michizuki, Jpn. J. Appl. Phys. 46, 601 (2007).

[26] J. Urata, M. Goldstein, M. F. Kimmitt, A. Naumov, C. Platt, and J.E. Walsh, Phys. Rev. Lett. 80, 516 (1998).

[27] R. Temkin, Science 280, 854 (1998).

[28] A. Bakhtyari, J. E. Walsh, and J. H. Brownell, Phys. Rev. E 65, 066503 (2002).

[29] O. H. Kapp, Y.-e Sun, K.-J. Kim, and A. V. Crewe, Rev. Sci. Instrum. 75, 4732 (2004).

[30] H.L. Andrews and C.A. Brau, Phys. Rev. ST Accel. Beams 7, 070701 (2004).

[31] H. L. Andrews, C. H. Boulware, C.A. Brau, and J.D. Jarvis, Phys. Rev. ST Accel. Beams 8, 050703 (2005).

[32] H. L. Andrews, C. H. Boulware, C. A. Brau, J. T. Donohue, J. Gardelle, and J. D. Jarvis, New J. Phys. 8289 (2006).

[33] V. Kumar and K. J. Kim, Phys. Rev. E 73, 026501 (2006). 
[34] G. F. Mkrtchian, Phys. Rev. ST Accel. Beams 10, 080701 (2007).

[35] H. L. Andrews, C. A. Brau, J. D. Jarvis, C. F. Guertin, A. O'Donnell, B. Durant, T. H. Lowell, and M. R. Mross, Phys. Rev. ST Accel. Beams (to be published).

[36] D. Li, K. Imasaki, X. Gao, Z. Yang, and Gun-Sik Park, Appl. Phys. Lett. 91, 221506 (2007).

[37] J. Gardelle and J.T. Donohue, IEEE Trans. Electron Devices 56, 769 (2009).

[38] H. L. Andrews, J. D. Jarvis, and C. A. Brau, J. Appl. Phys. 105, 024904 (2009).

[39] V. Kumar and K.-J. Kim, Phys. Rev. ST Accel. Beams 10, 080702 (2007).

[40] C. A. Brau, Free-Electron Lasers (Academic Press, San Diego 1990), pp. 197-204.

[41] E. T. Scharlemann, in Laser Handbook, edited by W. B. Colson, D. Pellegrini, and A. Renieri (North-Holland, Amsterdam, 1990), pp. 327-342.
[42] H. L. Andrews and C. A. Brau, J. Appl. Phys. 101, 104904 (2007).

[43] A. Gover and P. Sprangle, IEEE J. Quantum Electron. 17, 1196 (1981).

[44] J. Gardelle and J. T. Donohue, IEEE Trans. Electron Devices 56, 769 (2009).

[45] C. A. Brau, Modern Problems in Classical Electrodynamics (Oxford, New York, 2004), p. 345.

[46] V. Kumar and K.-J. Kim, in Proceedings of FEL 08, Gyeongju, Korea, http://accelconf.web.cern.ch/ AccelConf/FEL2008/papers/mopph004.pdf.

[47] J. A. Swegle, Phys. Fluids 28, 3696 (1985).

[48] B. Levush, T. M. Antonsen, A. Bromborsky, W.-R. Lou, and Y. Carmel, IEEE Trans. Plasma Sci. 20, 263 (1992).

[49] A. Gover and P. Sprangle, IEEE J. Quantum Electron. 17, 1196 (1981).

[50] I. J. Owens and J. H. Brownell, J. Appl. Phys. 97, 104915 (2005). 